\title{
Seniorenpolitische Perspektiven - Handlungsansätze für universitäre Forschung und Lehre
}

I.

Da eine anständige Rede mit einem Bescheidenheitstopos zu beginnen hat, möchte ich gleich zu Anfang meines Beitrags sagen, dass ich die aus seniorenpolitischer Sicht möglichen oder wünschenswerten Handlungsansätze für universitäre Forschung und Lehre in Bezug auf die Lebenslage Alter natürlich nicht umfassend darstellen kann wer könnte das schon?

Ich möchte vielmehr den Versuch machen, in Bezug auf den Handlungsrahmen hessischer Seniorenpolitik darzustellen, welche konkreten Zusammenhänge zwischen universitärer Forschung und Lehre einerseits und der seniorenpolitischen Programmund Konzeptentwicklung in Hessen andererseits bestehen. Von besonderem Interesse ist dabei natürlich die Frage, wo Defizite, also nicht genutzte Handlungsansätze, zu konstatieren sind.

Hinsichtlich der Frage, von welchem seniorenpolitischen Handlungsrahmen denn insoweit auszugehen sei, bin ich in der glücklichen Lage, auf die zu Anfang der laufenden Legislaturperiode herausgegebenen »Seniorenpolitischen Grundsätze und Leitlinien der Hessischen Landesregierung « verweisen zu können. Für Interessierte habe ich einige Exemplare mitgebracht.

Dieses Programm ist an dem Leitziel ausgerichtet, alten Menschen auch bei wachsendem Hilfebedarf eine möglichst selbständige Lebensführung zu gewährleisten. $\mathrm{Zu}$ diesem Zweck müssen Lebensräume entsprechend den Bedürfnissen und Möglichkeiten alter Menschen bewusst gestaltet werden, sowohl im häuslichen Bereich wie insbesondere auch im Heim. In ganz besonderem Maße gilt dies für die wachsende Zahl demenzkranker alter Menschen.

Diese Leitziele heben sich bewusst ab von der seniorenpolitischen Programmatik früherer Jahre, die noch viel stärker an einem - ich will nicht sagen fürsorgerischen, aber doch - fürsorglichen Denken ausgerichtet war. Systementwicklung in der Altenhilfe ging traditionell aus von im Verlauf des Alternsprozesses festzustellenden Hilfebedarfen, denen dann die Entwicklung der Hilfeangebote entsprechen sollte. Das hat natürlich bei zunehmender Hochaltrigkeit auch heute noch seinen Sinn, aber das Denken in der Kategorie von Lebensräumen, in denen auch und gerade schwer beeinträchtigte Menschen mit hohem Hilfebedarf mit relativer Selbständigkeit leben können: das ist eine andere Welt, was man z. B. am Unterschied zwischen einer sog. Hausgemeinschaft für Demenzkranke und herkömmlichen Pflegeheimen unschwer erkennen kann.

Fragt man nun, welche Bezüge zwischen diesem Parameterwechsel und universitären Aktivitäten bestehen, so denkt man in erster Linie an Frau Lehr und Herrn Kruse in Heidelberg, die viel zu einer adäquaten Weiterentwicklung des Altersbildes in der Gesellschaft und damit auch zur Weiterentwicklung seniorenpolitischer Programmatik beigetragen haben. In Hessen wäre hier in erster Linie an die Soziale Gerontologie in 
Kassel zu denken und natürlich an die Universität des dritten Lebensalters, die viel zur Erörterung seniorenpolitischer Fragestellungen nicht nur im Kreise derer beiträgt, die daran ein sozusagen biografisches Interesse haben.

Was ich mir wünschen würde, wäre, dass die »Lebenslage Alter « insgesamt stärker in den Mittelpunkt universitärer Aktivitäten rückt, und zwar jenseits fachspezifischer Fragen, so z. B.

- im Hinblick auf das Verhältnis zwischen den Generationen;

- im Hinblick auf die Frage nach einer »neuen gesellschaftlichen Solidarität« jenseits der Finanzierungsprobleme der sozialen Sicherungssysteme;

- im Hinblick auf »neue soziale Netzwerke« und Perspektiven des ehrenamtlichen oder bürgerschaftlichen Engagements, was angesichts des steigenden Anteils Alleinlebender besonders wichtig wäre;

- im Hinblick auf Möglichkeiten und Perspektiven interdisziplinärer Zusammenarbeit ausgehend von der Komplexität der Bedarfslagen im Alter.

\section{Nun einige Anmerkungen zu spezifischen seniorenpolitischen Handlungsfeldern:}

Beginnen wir unfreundlicherweise mit einem handfesten universitären Defizit: Hessen hat in Umsetzung des interdisziplinär angelegten Hessischen Geriatriekonzeptes in 10 Jahren weit über 1000 Geriatriebetten geschaffen - und hat keinen einzigen Lehrstuhl für Geriatrie. Hessische Kliniken müssen sich ihre Geriater aus anderen Bundesländern oder aus dem Ausland holen. Der an dieser Stelle übliche Hinweis auf die Hochschulautonomie ist kein Ruhmesblatt für hessische Universitäten. Auch eine Auslands-Gastprofessur für Geriatrie und Pharmakologie - so begrüßenswert sie ist kann hier nicht entscheidend weiterhelfen.

Allerdings muss ich zur Ehrenrettung hessischer Universitäten sagen, dass es im Rahmen hessischer Modellprojekte zur geriatrischen Behandlung und Rehabilitation eine langjährige, fruchtbare Zusammenarbeit mit der Universität Kassel gibt, genauer gesagt mit der vormaligen interdisziplinären Arbeitsgruppe für angewandte soziale Gerontologie, inzwischen aber umgewidmet in eine Forschungsgruppe Prävention und Rehabilitation innerhalb eines Fachbereichs, was allerdings Nachteile beim Zugriff auf Haushaltsmittel hat, wie ich mir habe sagen lassen.

Verbesserungsfähig ist wohl auch die Situation in der Gerontopsychiatrie. Da gibt es zwar seit kurzem die Stiftungsprofessur der BHF-Bank-Stiftung in Frankfurt, aber sonst ist, nach dem was ich in Erfahrung bringen konnte, der Stand der Entwicklung universitärer Angebote im Bereich der Gerontopsychiatrie in Hessen unbefriedigend. Dies mag u. a. auch mit der Frage zusammenhängen, in welchem Verhältnis die Gerontopsychiatrie zur Allgemeinen Psychiatrie einerseits und zur Geriatrie andererseits steht.

Allerdings sind im Bereich der Gerontopsychiatrie hochrelevante Strukturentwicklungen im Gange, so insbesondere der Aufbau eines gerontopsychiatrischen Verbunds im nordhessischen Raum, ausgehend und moderiert von der Klinik für Psychiatrie in Bad Emstal-Merxhausen, Herrn Dr. Struwe, der dort auch Wichtiges leistet im Bereich der Behandlung von Depressionen im Alter. Dieser gerontopsychiatrische 
Verbund führt klinische Gerontopsychiatrie incl. Institutsambulanzen, Beratung, Fortund Weiterbildung, Supervision und Krisenintervention sowie ambulante und stationäre Pflegeeinrichtungen zu einem Gesamtkonzept zusammen - ein Prozess, den ich mir für ganz Hessen wünschen würde. Die Frage wird erlaubt sein, welche Funktion Universitäten in einem solchen Prozess übernehmen könnten.

Festzustellen ist in diesem Zusammenhang außerdem, dass Impulse für die Konzeptentwicklung im Bereich der Betreuung und Pflege Demenzkranker, so insbesondere was die sog. Hausgemeinschaften angeht, in Hessen bisher eher von den Fachhochschulen ausgegangen sind, so z. B. über die wissenschaftliche Begleitung des Projekts »Heimverbundene Hausgemeinschaft für Demenzkranke« in Wetter durch die Fachhochschule Frankfurt, was vermutlich mit der Ansiedlung pflegewissenschaftlicher Studiengänge auf Fachhochschulebene zusammenhängt.

Bei der Befassung mit solchen Konzepten ist mir allerdings geradezu schlagartig deutlich geworden, dass auf dem Weg über Hausgemeinschaftskonzepte, wie neuerdings auch sog. Wohngemeinschaften für Demenzkranke, der Gedanke des Zusammenlebens alter Menschen in Gruppen überhaupt erstmals Eingang in die Konzeptentwicklung der Altenhilfe findet. Konzepte auf pflegewissenschaftlicher Grundlage gehen, soweit ich das überblicken kann, nach wie vor in erster Linie von der bilateralen Interaktion Pflegender - Pflegebedürftiger aus, und auch die Gerontologie hat sich bisher - was mir Herr Kruse vor kurzem in einem persönlichen Gespräch bestätigt hat - um das Konstrukt der Gruppe wenig gekümmert. Ich interpretiere das so, dass das der Forschung in diesem Bereich zu Grunde liegende Altersbild nach wie vor vom sozusagen »bürgerlichen « Individuum ausgeht, das seinen privatpersönlichen Lebensbereich (so es denn ein Einzelzimmer hat!) nur zu bestimmten Anlässen (Mahlzeiten, Programmangebote) verlässt, im Übrigen aber alleine lebt und dabei gelegentlich Besuch empfängt. Für welche Menschen mit welcher Befindlichkeit das enge Zusammenleben in einer Gruppe mit gemeinsamen Aktivitäten über den Tag hinweg geeignet ist, bedarf noch genauso der Klärung wie die Frage, welche tagesstrukturierenden Elemente jenseits hauswirtschaftlicher Verrichtungen für Menschen mit oder auch ohne dementielle Erkrankungen beim Zusammenleben in einer Wohn- und Pflegegruppe in Frage kommen. In diesem Zusammenhang wäre auch von Interesse, welche Möglichkeiten des Verstehens des Verhaltens demenzkranker alter Menschen erschlossen werden können und welche Schlussfolgerungen sich daraus für die Konzeptentwicklung ergeben. Sehen Universitäten in solchen Fragen auch Aufgaben, die sie selbst etwas angehen?

Durchaus Erfreuliches von der Zusammenarbeit mit hessischen Universitäten kann ich berichten von der Entwicklung des Heimbaus, zu der hessische Hochschullehrer, insbesondere aus Darmstadt, als Architekten Wesentliches beitragen. Ähnliches ist zum Thema »Seniorengerechte Entwicklung industrieller Produkte « zu sagen. Hier hat eine Arbeitsgruppe unter der Federführung unseres Hauses und mit engagierter Beteiligung der Universität Darmstadt und der Fachhochschule Frankfurt wichtige Beiträge zur Bewusstseinsbildung und durchaus auch zur Verbreitung seniorengerechter Produkte am Markt mittels entsprechender Informationsbroschüren und -veranstaltungen geleistet. 
Nun ein Blick auf die Rechtsentwicklung in Bezug auf die Lebenslage Alter. Betrachtet man den seniorenpolitischen Handlungsrahmen, in dem wir uns als Land bewegen, daraufhin, welche Rechtsgrundlagen das deutsche Sozialrecht bereithält, dann stoßen wir zunächst auf den zweifellos durchnormierten - manche sagen überregulierten - Bereich der Pflegeversicherung. Darüber hinaus gibt es eigentlich nur $\S 75$ BSHG (Altenhilfe) als sedes materiae für Hilfen, Dienste und Einrichtungen im Alter.

Dieser Sachverhalt ist allein schon eine Diskriminierung der Lebenslage Alter, denn kann man vor dem Hintergrund der demographischen Entwicklung heute wirklich noch sagen, dass Alter eine »besondere Lebenslage« ist, die rechtlich und gesellschaftspolitisch in die Sozialhilfe gehört? Im Übrigen hat $§ 75$ BSHG in der Rechtspraxis und in den Leistungsstatistiken der Sozialhilfe bis heute so gut wie keine Bedeutung gehabt.

Diese Einschätzung hat - neben anderen Motiven - zu dem Gesetzgebungsvorhaben eines Bundesaltenhilfegesetzes oder auch Altenhilfestrukturgesetzes geführt, das immerhin in der letzten und in der laufenden Legislaturperiode Eingang in das Regierungsprogramm der Bundesregierung gefunden hat. Kernstück dieses Gesetzgebungsvorhabens, das ich aus eigener Mitarbeit in einer früheren Projektgruppe des Bundesseniorenministeriums gut kenne, ist die Ausrichtung aller Hilfen auf das Leitziel größtmöglicher Autonomie und Selbständigkeit in der Lebensführung. Instrumenteller Schwerpunkt eines solchen Gesetzes wäre die Einführung einer personenbezogenen Hilfeplanung oder auf gut neudeutsch Case-management.

Dabei handelt es sich um ein international bewährtes und anerkanntes Instrumentarium für die Anlage und Umsetzung von Hilfekonzepten im Bereich sozialer Dienste für Menschen mit komplexem Hilfebedarf. Sie sind bisher in je unterschiedlicher Weise etabliert und zum Teil auch verrechtlicht, z. B. in

- der Kinder- und Jugendhilfe;

- der Rehabilitation und Teilhabe Behinderter;

- der Bewährungshilfe;

- der Beschäftigungsförderung;

- im Gesundheitswesen.

Gibt es hinreichende Gründe, Case-management im Sinne personenbezogener Hilfeplanung in der Altenhilfe nicht einzuführen?

Die Perspektive eines solchen Gesetzgebungsvorhabens ist allerdings ungewiss, denn die Anforderungen an die Gesetzgebungsbefugnis des Bundes im Bereich der konkurrierenden Gesetzgebung durch Art. 72 Abs. 2 GG sowie mögliche Überschneidungen mit der kommunalen Selbstverwaltung, u. U. auch mit der Gewerbefreiheit, stellen Hindernisse auf dem Weg der Gesetzgebung dar. Hier stellt sich mir die Frage, welche Beiträge Universitäten jenseits vereinzelter Gutachten, die die Bundesregierung in Auftrag gibt, zur Weiterentwicklung des Rechts der Altenhilfe leisten könnten.

III.

Ich habe versucht, ohne Anspruch auf Vollständigkeit, an einigen Beispielen zu illustrieren, welche Wechselbeziehungen zwischen der seniorenpolitischen Konzeptent- 
wicklung in Hessen und Aktivitäten hessischer Universitäten in meinem Blickfeld sind. Dass ich mich über deren perspektivische Weiterentwicklung freuen würde, liegt auf der Hand. Voraussetzung hierfür wäre einerseits eine intensivere fachliche Zusammenarbeit von Institutionen und Organisationen der Altenhilfe einschließlich des zuständigen Ressorts der Landesregierung mit den entsprechenden Organisationseinheiten der Universität in Bezug auf spezifische Fragestellungen und Problemfelder. Zum Anderen stelle ich mir einen interdisziplinären Diskurs über die Lebenslage Alter vor, der über fachspezifische Probleme hinaus die Frage in den Mittelpunkt stellt, wie wir im Alter leben wollen; eine Frage, die nicht nur diejenigen etwas angeht, die heute schon alt sind oder dem in Kürze entgegensehen, denn Lebensräume, die wir im Alter haben wollen, müssen heute erdacht, geplant und ausgestaltet werden, und dies in immer schärfer werdender Konkurrenz zu anderen Politikzielen. Ich bin überzeugt davon, dass das »Forum Alterswissenschaften und Alterspolitik «, das aus Anlass und auf Initiative von Gisela Zenz konstituiert werden soll, hierzu einen wesentlichen Beitrag leisten wird. Ich würde mich freuen, wenn ich dabei ein wenig mithelfen könnte. 